\title{
Knowledge of Pregnant Mothers on the use of Prevention of Mother to Child Transmission of HIV Services in Kenya: A Cross Sectional Study at Bungoma County Referral Hospital
}

\author{
Article by Taratisio Ndwiga \\ PhD in Public Health, Texila American University \\ E-mail: taratisiondwiga@texilaconnect.com
}

\begin{abstract}
Background: Mother-to-child transmission (MTCT) of the human immunodeficiency virus (HIV) remains the major source of HIV infection in young children. Targeting pregnant women attending antenatal clinics provides a unique opportunity for the implementing prevention of mother-to-child transmission (PMTCT) programs against HIV infection of newborn babies.

Objective: To identify factors affecting Knowledge of prevention of mother to child transmission of HIV services among pregnant women attending antenatal clinic in Bungoma County Referral Hospital.

Methodology: A cross-sectional study was conducted among pregnant mothers attending antenatal care clinic at Bungoma County Referral Hospital. A systematic random sampling technique was used to select 50 respondents, using Fisher et al, 1999. The data was collected using structured interviewer administered questionnaire prepared to address knowledge, and associated factors on PMTCT services. Data was entered and analyzed using Statistical Package for Social Sciences (SPSS) version 20.

Findings: A big number (40\%) of the mothers did not know about PMTCT services which were being offered in the hospital. It was noted that the respondents who had little or no knowledge of the services were mostly below 25 years of age and those with low education levels. On the attitude towards PMTCT services it was found that (44\%) reported that it is good to take on PMTCT services if reactive and (56\%) of respondents reported that PMTCT services are wastage of time because AIDS has no cure.

Conclusion: The educational level was the most important factor in understanding PMCTC services

Recommendation: To improve and increase sensitization and awareness to the communities on what PMTCT program entails.
\end{abstract}

Keywords: Prevention of mother to child transmission, Human immunodeficiency virus, Acquired immunodeficiency syndrome, Antenatal clinic.

\section{Introduction}

Mother-to-child transmission (MTCT) is when an HIV-infected woman passes the virus to her baby. This can occur during pregnancy, labor and delivery, or breastfeeding. Without treatment, around 15-30\% of babies born to HIV positive women will become infected with HIV during pregnancy and delivery. A further 5-20\% will become infected through breastfeeding [1]. In 2009, around 400,000 children below 15 years became infected with Human immunodeficiency virus [2], almost all of MTCT infections occurred in Sub-Saharan Africa, and more than $90 \%$ were as a result of mother-to-child transmission (MTCT) during pregnancy, labor/ delivery, and breastfeeding. There is still fear of disclosing one's HIV status(or of learning it) because of the stigma that exists for people leaving with HIV/AIDS which hinders women from seeking PMTCT services and results in poor adherence to PMTCT interventions, in particular safer infant-feeding decisions. Being confident enough to disclose one's HIV status is one of the most powerful ways to reduce HIV related stigma. Disclosing one's status also has other benefits, it can encourage partners to be tested for HIV and prevent the spread of HIV by allowing those infected to openly take appropriate prevention steps. Therefore knowledge and awareness on PMTCT of HIV has an impact on the practice of HIV testing, taking medication and attitude toward PMTCT interventions among pregnant mothers. Globally, approximately 289,000 maternal deaths occurred in 2013. Sub-Saharan Africa 
Texila International Journal of Public Health

Volume 5, Issue 2, May 2017

accounts for about $98 \%$ of maternal deaths in Africa. In addition the human immunodeficiency virus (HIV) and the acquired immune deficiency syndrome (AIDS) were associated with 1.8 million deaths including 250,000 among children less than 15 years in 2010 [3]. In Cameroon, AIDS-related diseases were associated with 980 maternal deaths in 2010 meanwhile the same year, 5\% ofunder-5 mortality was linked to HIV/AIDS [4]. Maternal and child health outcomes have been shown to be linked to the quality of antenatal care (ANC), delivery services and post-natal care [5]. Prevention of mother-to child HIV transmission (PMTCT) strategies introduced in 2004 have become integrated into all maternal and child health services. Antenatal care (ANC) is a major entry point for PMTCT programs especially in countries with a high prevalence of HIV. The World Health Organization (WHO) recommends four ANC visits during pregnancy [6], which allows for early diagnosis, prevention or treatment of conditions which may jeopardize the health of the mother or her unborn baby. High maternal, neonatal and child mortality rates are associated with inadequate and poor-quality maternal health care, reflected in the "three delays"; deciding to seek care, reaching the health care facility in time and receiving adequate treatment [4] . In the absence of intervention $50 \%$ of children born to HIV positive mothers will die before reaching their second birthday [7]. The challenges facing the PMTCT programs in sub-Saharan Africa are numerous including large proportions of home deliveries, fear of the cultural implications of a positive HIV test result, such as the lack of male partner support and even violence [8]. In the context of an HIV epidemic, the WHO developed a four-prong approach to optimize the effectiveness of PMTCT interventions aimed at improving maternal and child health, which include primary prevention of HIV among women of reproductive age, prevention of unintended pregnancies among HIV infected women, PMTCT interventions and provision of care, treatment and support for children, mothers and affected families.

The expected expansion of PMTCT services on the Sub-continent has however, been faced with less than satisfactory utilization. Use of services such as facility delivery and postnatal prophylactic ARV is reported to be between $20 \%$ and $47 \%$ in many settings [9].

In Kenya, interventions to reduce PMTCT are integrated in Maternal and Child Health (MCH) services in health service delivery institutions. These also include HIV testing during ANC attendance to identify pregnant women living with HIV, maternal and infant antiretroviral treatment, safe obstetrical care, infant feeding counseling and support, and family planning services to delay future pregnancies or prevent unintended pregnancies [10]. Kenya launched its PMTCT program in 2000, but only modest expansion of PMTCT services occurred in the first three years that followed National Aids and STI. Despite these low levels of service uptake, there have been limited studies attempting to document reasons for underuse of PMTCT services. The objective of this study was to determine factors affecting utilization of PMTCT services among pregnant women as well as their perceptions of service delivery dynamics that are associated with the uptake of PMTCT services.

In a setting like Kenya with ethnic diversities, many factors can affect a woman's ability to seek MCH and PMTCT services and hence adopt positive behaviors. These may include marital status, socioeconomic status, age, parity, history of delivery complications, religion and cultural factors.

Identifying factors influencing uptake of MCH/PMTCT will inform policies and programs to help improve maternal and child health outcomes. The cascade for diminishing services used at

PMTCT/MCH services demonstrate that Kenya is and may continue to face key public health challenges for $\mathrm{MCH} / \mathrm{PMTCT}$. Considering national and global interest in the millennium sustainable goals 4 (reduce child mortality), 5 (improve maternal health) and 6 (combat HIV/AIDS, malaria, tuberculosis and other diseases) understanding these factors is crucial. Also knowledge of barriers will ensure that women and children are retained in prevention, treatment and care services in Kenya.

Despite improvements in PMTCT services over the years, MTCT of HIV infections is high especially in sub-Saharan Africa. In 2009 alone around 400,000 children less than 15yrs became infected with HIV and 1.3 million children and adults died of [2]. Mother-to-child transmission (MTCT) of human immunodeficiency virus (HIV) remains the major source of HIV infection in young children. Targeting pregnant women attending antenatal clinics provide a unique opportunity for implementing prevention of mother-to-child transmission (PMTCT) programs against HIV infection of newborn babies. The objective 
was to investigate the pregnant women's knowledge of Prevention of Mother- to-Child Transmission of HIV (PMTCT) services.

\section{HIV/AIDS and PMTCT in kenya}

Kenya began implementing PMTCT programs as standalone programs in 2002 and has committed itself to elimination of mother-to-child HIV transmission by 2015. Global elimination of mother-to-child HIV transmission (MTCT) is targeted for 2015 and is an initiative which will require strategic improvements in service delivery [11]. As PMTCT interventions have expanded globally, challenges in delivery and uptake of services have persisted. The World Health Organization (WHO) estimated that in 2010, in low and middle income countries, only 35\% of pregnant women received HIV testing, and less than half of HIV infected women tested accessed antiretroviral (ARVs) for PMTCT. The Kenya Ministry of Health has made great strides to increase PMTCT coverage, with provision of ARVs to HIV-infected pregnant women increasing from $20 \%$ uptake in 2005 to $69 \%$ in 2011 [12]. As a result, new child HIV infections in Kenya have decreased, averting 46,000 new infections since the introduction of PMTCT [13]. However, the number of newly infected children per year remain high (13,000 in 2012), [3] and recent estimates of MTCT rates, while 5\% in clinical trial settings, range from 8 - 27\% in Kenyan surveys [14].

PMTCT programs in Kenya include HIV counseling and testing, referral to HIV care/treatment for those found positive, provision of prophylactic ARV medication to HIV positive mothers before delivery and for infants within 72 hours of birth, infant feeding counseling and DNA polymerase chain reaction (PCR) testing for infants born to HIV positive mothers [15].

The HIV status of infants born to HIV positive mothers are detected as early as 6 weeks after birth using the polymerase chain reaction (PCR) test. The test can reliably and accurately detect HIV DNA on a dried blood spot (DBS) specimen. The early infant diagnosis has been improved due to the DNA/PCR test and can subsequently lead to early referral of HIV infected infants for appropriate care and treatment and this reduces the morbidity and mortality of HIV positive infants/children. Without any intervention, statistics show, $30 \%$ of HIV infected children will die in the first year of life and $50 \%$ would be dead by the second year [12].

\section{Factors affecting PMTCT utilization}

Generally, as documented by Health Bridge in 2007, the PMTCT factors affecting many African countries include: home delivery, involvement of males, compliance to taking ARV's and prophylaxis and follow-up, stigma and feeding choices, costs and drug availability, human resources and health system factors and PMTCT data management.

The Namibia PMTCT country report which was compiled by [15] outlined the key implementation challenges to scaling up PMTCT program. Some of the key challenges included factors such as the lack of male involvement, shortages of staff and neglected follow-up after delivery for both mother and baby. Infant feeding poses another challenge as little is known what will happen when the mother leaves the health facility. Training and retraining of health care workers, is another challenge as they are continuously on the move in search of careers. Also there is a weak link when it comes to comprehensive care for mother and baby, children and partner. Sometimes it is difficult to tell that if someone tests positive at ANC they will end up at ART clinic after being referred and also at times there are great distances between ANC and ART clinics and people must travel to an ART clinic and some may not go due to affordability. It was also documented in this report that from 62,000 expected pregnancies each year in Namibia only 55,000 of them attend ANC and about 7,000 pregnant mothers are not captured. 
Texila International Journal of Public Health

Volume 5, Issue 2, May 2017

\section{Methodology}

\section{Location and study design}

Bungoma County Referral Hospital is located within Bungoma town in Bungoma South Sub- County. A descriptive cross sectional study was conducted to collect data. The study period was from December $5^{\text {th }}$ to $23^{\text {rd }}, 2016$. Systematic random sampling was used to select the respondents. A sample size was determined using [16] formula, which gave the final sample size of 50 respondents.

In calculating sample size; the level of utilization is $50 \%$

$$
\begin{aligned}
& \text { Thus } p=50 \% \\
& \text { And } q=50 \%
\end{aligned}
$$

From fishers formula $n_{o}=\left(z^{2} P Q\right) /\left(d^{2}\right)$

$z=1.96$ (z score associated with $95 \%$ confidence level.

$d=$ discrepancy tolerated on $p=0.1$ (so the level of level of utilization is given a \pm of 0.1 )

Therefore, $n_{o}=\left(1.96^{2} * 0.25\right) /(0.01) \approx 96$

Adjusting for a small target population of 105 target population;

$$
\begin{aligned}
& n=\left(N o^{n e}\right) /\left(N+n^{o}\right) \\
& (105 * 96) /(105+96) \approx 50.15
\end{aligned}
$$

Sample size $=50$.

\section{Data collection and analysis}

The data was collected using structured interviewer administered questionnaire prepared to address knowledge and associated factors on PMTCT services. The questionnaires were administered to all the systematically selected pregnant women who fulfilled the inclusion criteria while attending ANC clinic at the hospital. Data was entered into and analyzed using Statistical Package for Social Sciences (SPSS) version 20 .

\section{Ethical consideration}

Research Ethical approval was sought from Bungoma County Referral Hospital administration committee; all selected respondents were communicated to, about the objective of study in order to obtain their verbal consent before administering questionnaires. Information communicated with individual subjects was kept private and confidential.

\section{Study findings}

\section{Introduction}

This section presents the results on Socio-demographic information (Age, marital status, Educational level and occupation), Knowledge of PMCTC, sources of information and importance of HIV testing during pregnancy, as outlined below. 


\section{Socio-demographic variables}

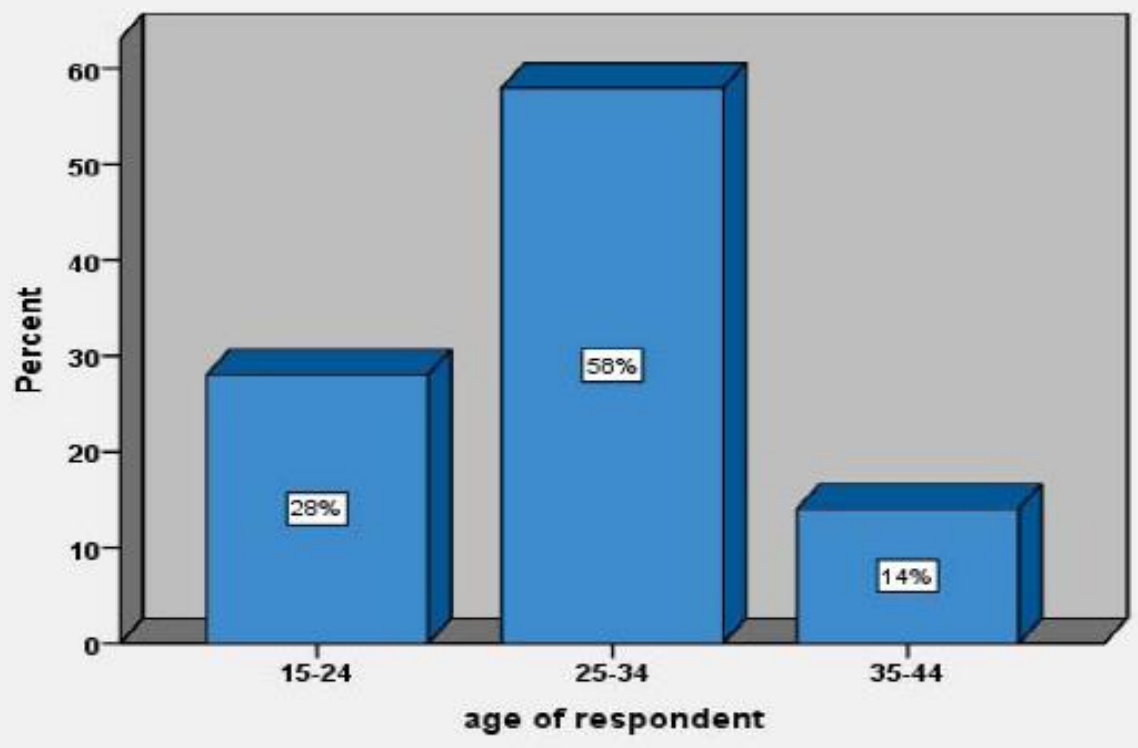

Figure 1. Age of respondents

Slightly more than half of the respondents (58\%) were aged 26-34 years while 28\% were aged between 15-24 years and 14\% were aged 35-44 years (figure 1).

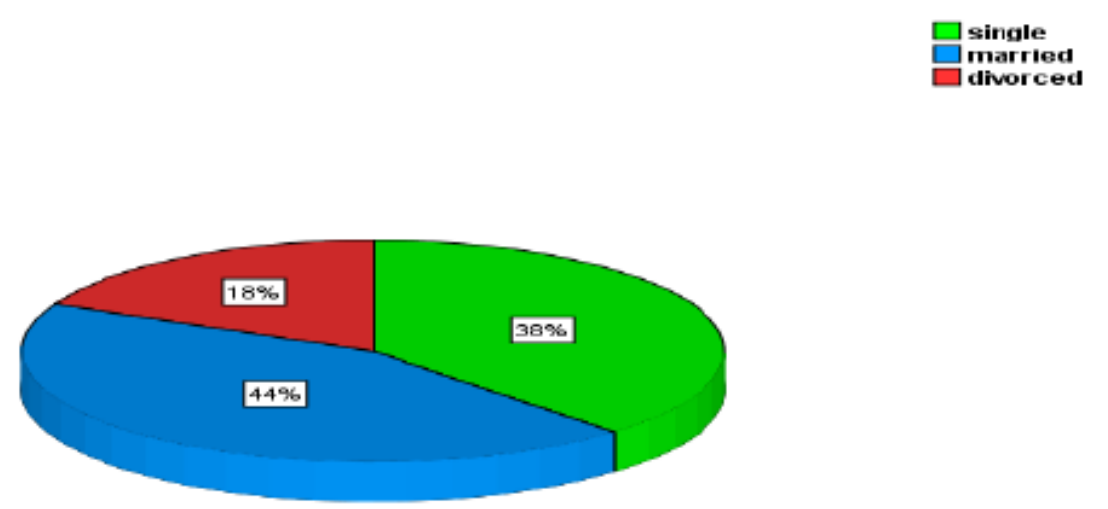

Figure 2. Marital status of the respondents

From the pie-chart, Majority (44\%) of the respondents were Married and $38 \%$ single while $18 \%$ divorced (figure 2 above). 


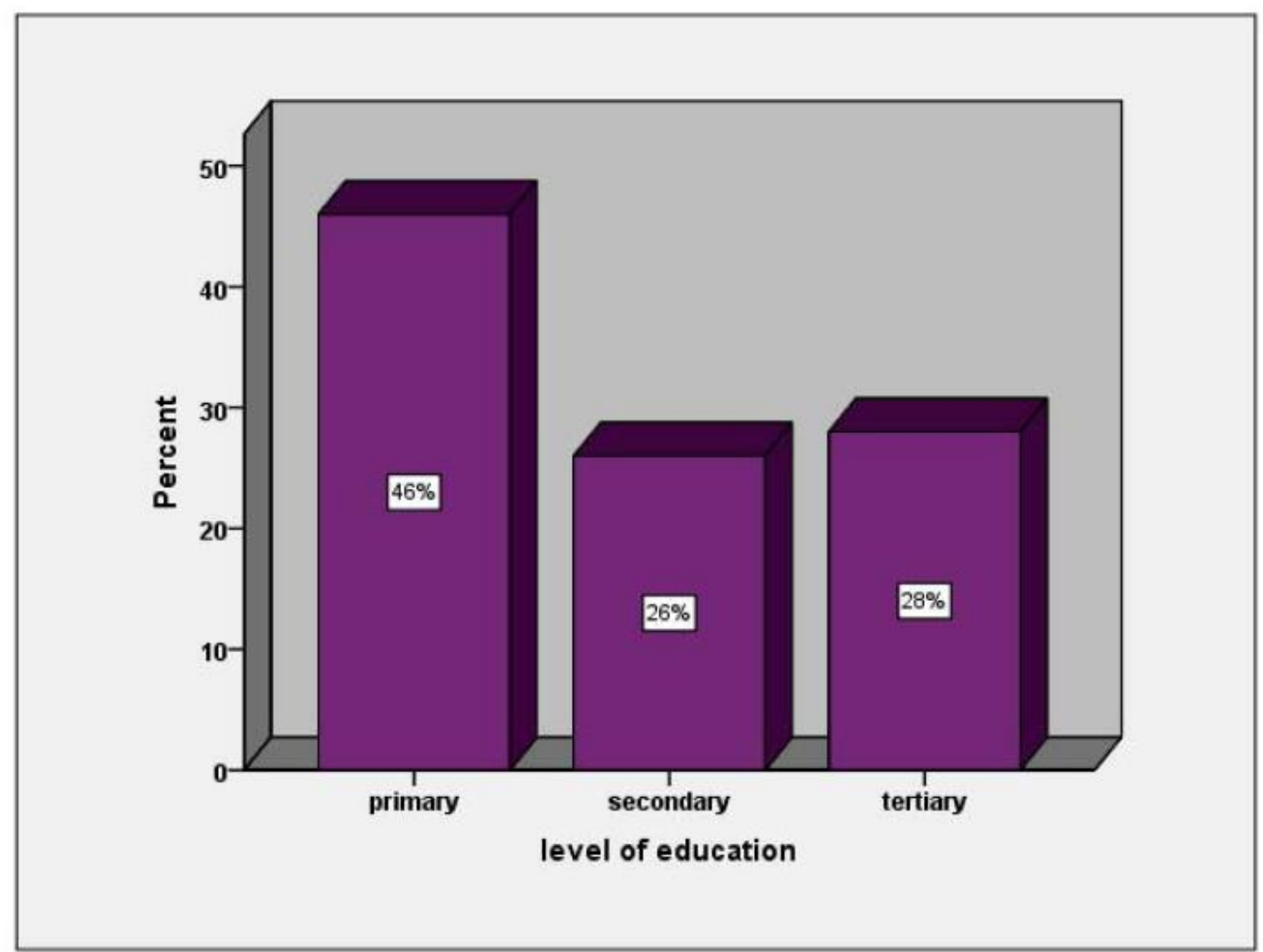

Figure 3. Level of education

From figure 3 above, most (46\%) of the respondents had attained primary education while (28\%) had attained tertiary education and $26 \%$ had attained secondary level of education.

Table 1. Occupation of the respondents

\section{Knowledge ON PMTCT}

\begin{tabular}{|l|l|l|}
\hline Occupation & Frequency & Percentage \\
\hline Business & 19 & 38 \\
\hline Employed & 11 & 22 \\
\hline Farmer & 12 & 24 \\
\hline Students & 7 & 14 \\
\hline Housewife & 11 & 22 \\
\hline
\end{tabular}

Table 2. Knowledge on PMTCT

\begin{tabular}{|l|l|l|}
\hline & Frequency & Percentage \\
\hline Yes & 30 & 60 \\
\hline No & 20 & 40 \\
\hline
\end{tabular}

Table 2 shows respondents occupation from Business to house wife. The most of respondents were doing their own businesses while the least were the house wives. The employed constituted only $22 \%$.

PMTCT services were well known by a greater percentage of the respondents $60 \%$ while a smaller portion $40 \%$ didn't have knowledge on the same (Table 2). 


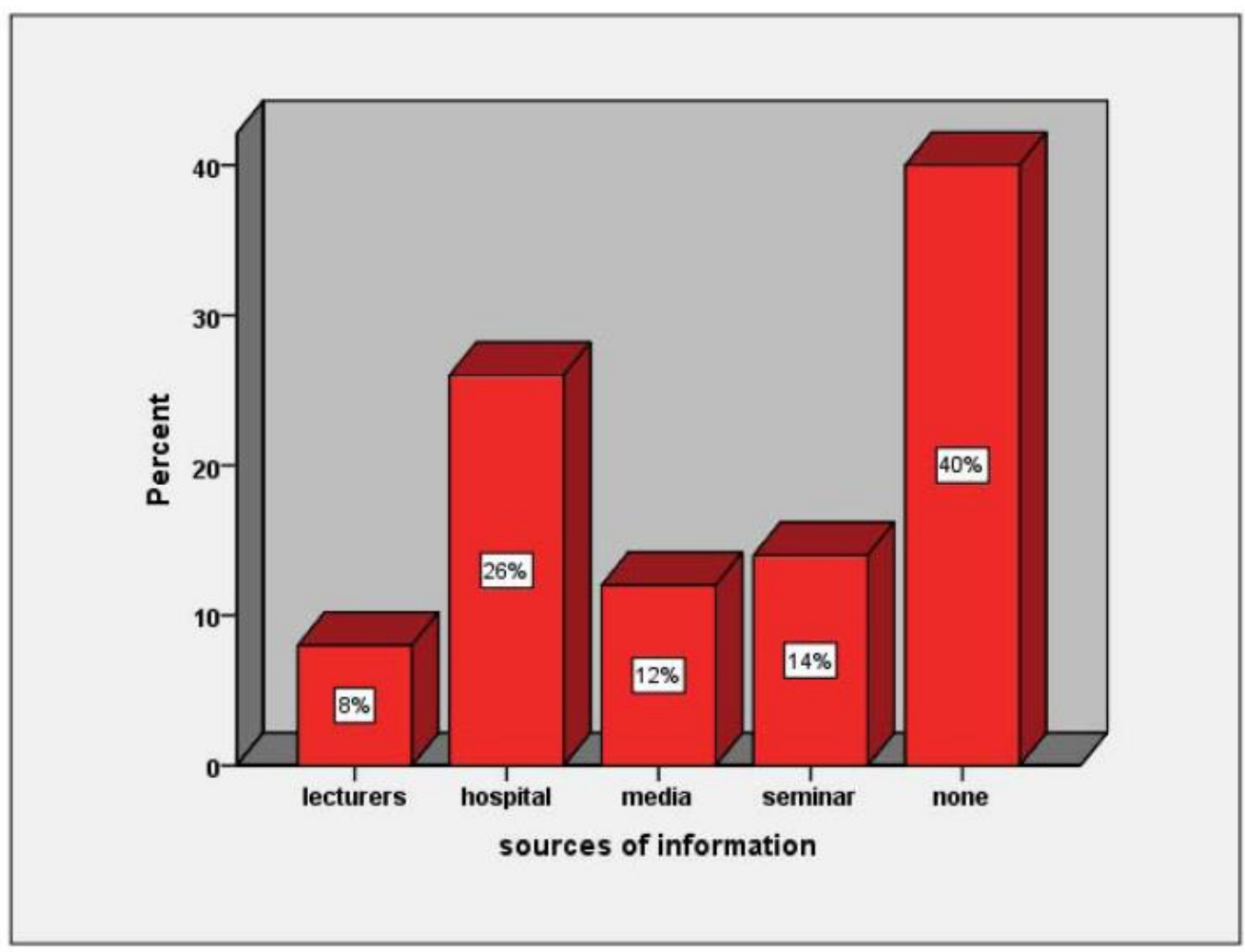

Figure 4. Source of information on PMTCT

Sources of information on the available PMTCT services included Hospitals (26\%), Lectures (8\%) Media (12\%), Seminars (14\%) while who had never heard of PMTCT service had no source of information accounting for $40 \%$ (figure 4 ).

Table 3. Importance of having HIV test during pregnancy

\begin{tabular}{|l|l|l|}
\hline & Frequency & Percentage \\
\hline Yes & 31 & 62 \\
\hline No & 19 & 38 \\
\hline
\end{tabular}

Majority of the respondents $62 \%$ knew that it was important to have a HIV test during pregnancy while $38 \%$ didn't know (Table 3).

\section{Attitude towards uptake of PMTCT services}

On whether the reactive women should have children due to fear of vertical transmission, a greater percentage of the respondent didn't have an idea $26 \%$ and those who said No were $36 \%$ while those who knew that reactive women should never breast feed their babies due to fear of mother to child transmission of HIV were $56 \%$.

A greater percentage $48 \%$ of the respondent spent too much time waiting for ANC services and a few $4 \%$ of them had no comment on time spent while majority of the respondent $52 \%$ spent too much time waiting for pmtct services, hence perceived the services as a waste of time 


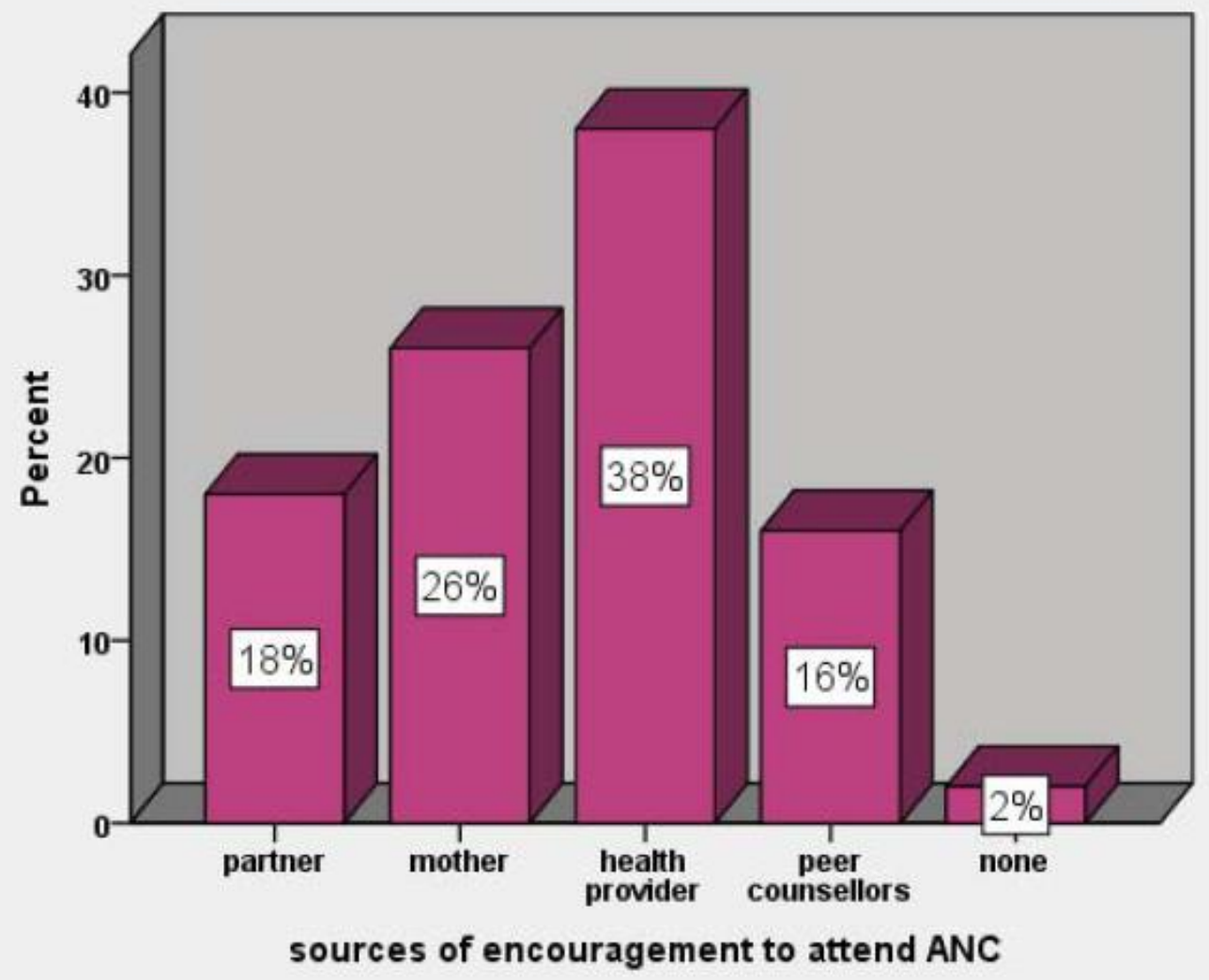

Figure 5. Sources of encouragement to attend ANC

Health providers $38 \%$ and mothers $26 \%$ played a major role on the source of encourage to pregnant mother to attend ANC while partner was $18 \%$, followed by peer counsellors $16 \%$ and none $2 \%$ ( Figure $5)$. 


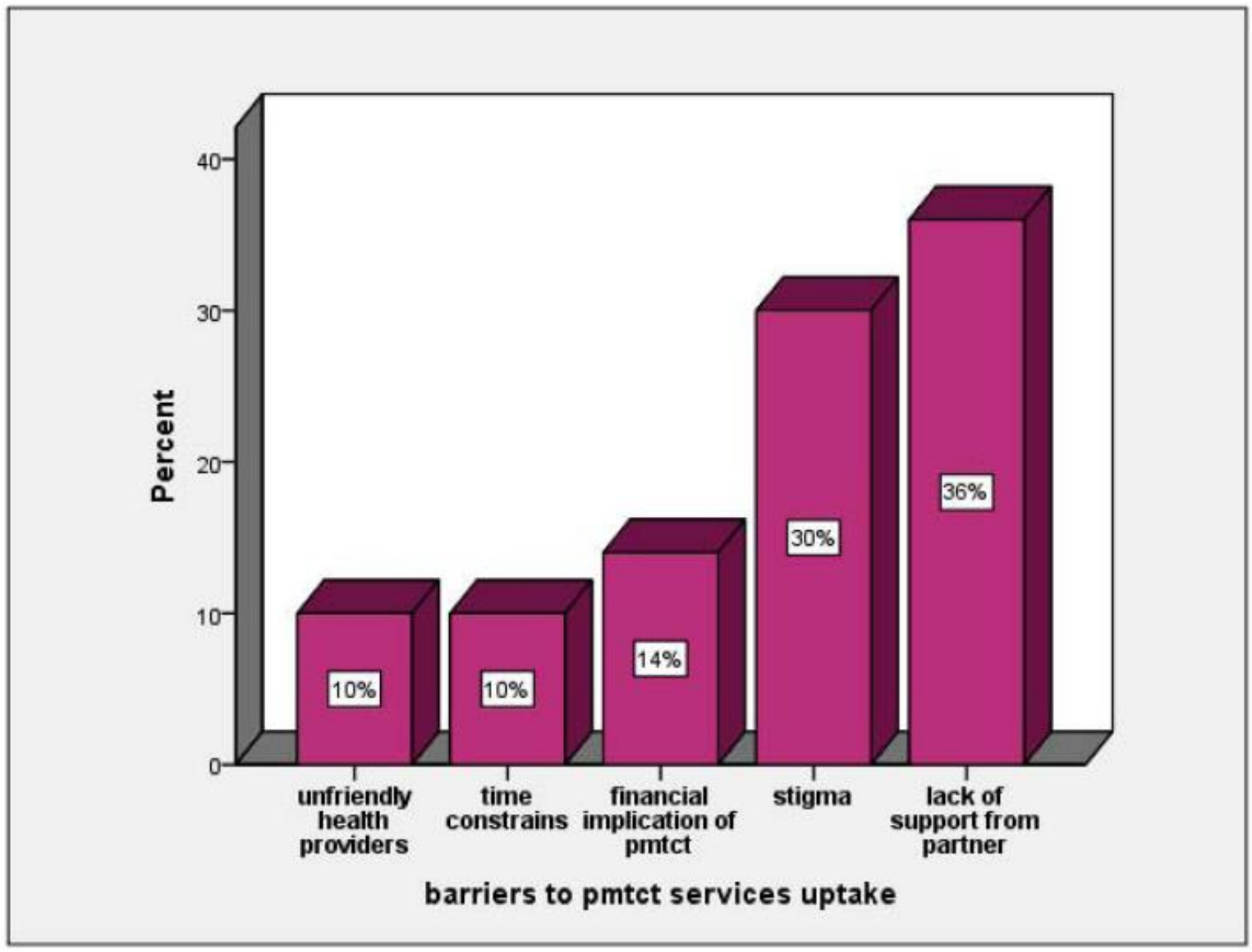

Figure 6. Barries to uptake of PMTCT services

Lack of support from partners and stigma were the main challenges to uptake of the available services (figure 6).

Majority of the respondents $58 \%$ were not comfortable and $42 \%$ were on the way of counseling, while pregnant women interviewed $56 \%$ were not willing and $44 \%$ were willing to take the services after testing due to stigmatization.

Majority of the respondents $68 \%$ were willing and $32 \%$ were not willing to have a HIV test after counseling while pregnant women interviewed $82 \%$ were not willing while $18 \%$ were willing to disclose their HIV status to anyone after testing.

\section{Discussion}

\section{Knowledge on PMTCT}

Bungoma County Referral Hospital MCH clinic serves a wide range of clients whose level of education ranges from primary to tertiary due to its location in an urban area. The respondents interviewed in the study had various levels of education ranging from primary to tertiary, with the majority (28\%) having tertiary, 26\% secondary and primary $26 \%$ level of education, however only 30 $(60 \%)$ of the respondents had knowledge on PMTCT. Majority of the respondents 31(62\%) had knowledge HIV as they were more common because most of continued health education provided by the hospital as well as the community health. The study showed those who were 26 years and above were more exposed to different sources of information and had experience on antenatal issues since they always came for counseling in Health Centre. The other groups were those who marry when they are still young and therefore do not take antenatal issues serious and the under 25 years who get pregnant before marriage and do not disclose due to fear from their parents and the society who are against having 
Texila International Journal of Public Health

Volume 5, Issue 2, May 2017

children out of marriage. These findings coincided with the studies previously found at Kakamega, Nakuru, Karatina and Thika district Hospitals in Kenya [9]. The study findings showed that respondents' occupation and the knowledge on PMTCT services revealed that those in formal employment had more knowledge on the services. The high level of knowledge about PMTCT by civil servants is attributed to their high education levels compared to other categories. It was also found that the same group complained about the much time they spend waiting for the antenatal services, since they always have to report back to the work places. Those involved in business, and other occupations were the next big category who knew about PMTCT services. This group was also exposed to different media and they also interact with different levels of people when they are carrying out their activities. Similar findings have been reported in a study done in Mbale regional hospital ANC [17]. Sources of information about PMTCT included interactions with friends during antenatal visits $26 \%$, media programs $12 \%$ and seminars lectures $8 \%$. It was also clear that the most reliable source among these was sensitization and counseling sessions at the hospital during antenatal visits.

\section{Attitude(s) towards PMTCT services}

Most pregnant mothers $62 \%$ felt it was important to have an HIV test and know their status while few (38\%) did not see the importance of knowing the HIV status because, as pregnant women had have a negative attitude towards HIV testing. The reason for this being fear and stigma associated with HIV/ AIDS as a disease. This indicates fear and stigma people have towards HIV and AIDS and the way People living with HIV/AIDS (PLWHA's) are stigmatized. However this is not only in Kenya but was also reported in Zambia in a study on PMTCT conducted by [18] whereby the findings indicated that there is a high level of stigma against HIV/AIDS patients. The community tends to shun persons who are known to be reactive or have symptoms of AIDS. Those who said it was good to have a HIV test during pregnancy was as a result of to increased health education and sensitization during ANC visits as well as increased dissemination of information on HIV.

Most of the mothers (36\%) reported that reactive woman should not have a baby while $26 \%$ of the respondents didn't have an idea and the rest $38 \%$ had knowledge on the same. Those who reported that a reactive woman should not have a baby gave a reason that this could shorten her already 'numbered days' since women loses a lot of blood in the delivery process. The other reason given was that some thought it was obvious a reactive woman will infect the baby, therefore they were not convinced there was need for the woman to give birth. This discussion was found similar to a study done at Mbale regional hospital ANC, indicating that there was still a gap in knowledge as far as PMTCT is concerned [17].

On the other hand respondents who reported that a reactive woman should have a baby recommended it because few of them are aware the baby can be born free of HIV because of PMTCT services, if the woman attended antenatal regularly and delivered from the hospital. Surprisingly, the other respondents looked at it in the cultural perspective that, it is the tradition for every woman to produce children irrespective of the HIV status.

Some mothers (20\%) said reactive woman should breastfeed her baby, while $56 \%$, said no and $24 \%$ didn't know if they should breastfeed their children if found reactive. The respondents who reported that reactive mother could breastfeed, at least knew that the baby born of an infected mother could still be safe if she is enrolled for PMTCT and also breastfeeding may not be dangerous when done exclusively, which was not known by some people that a reactive mother could breastfeed exclusively and the baby be safe. It was again found that among those who reported that the baby should be breastfed thought it was every mother's obligation to breast feed her baby. Others reported that there were mothers who could not afford to buy other types of milk, so breast milk was their only option. Among these however, there were those who knew about exclusive breastfeeding due to counseling from PMTCT program at the antenatal clinic.

On the issue of the husbands' and community's reaction when a wife/mother does not breastfeed her child, it was found out that if the husband knew the wife was sick, the wife/mother was advised by the health workers not to breastfeed at all then the husband supported her, but if the husband did not know, 
then this could cause problems at the family level. These findings were similar with those found by in a study done in Uganda and Tanzania [19].

Most of the respondents (48\%) reported spending too much time waiting for ANC services while a few (4\%) did have any comment on the same. This was as a result of high population in the town that led to high flow of clients at the clinic hence long waiting hours.

\section{Utilization of PMTCT services and associated barriers}

Study findings showed that there were a number of things that affected the level of utilization of

PMTCT services; these include lack of male involvement which counted for 36\%. [20] reported that many health facilities implementing PMTCT programs do not focus on involving men in these activities. This has led to poor communication between spouses as far as disclosure of HIV status is concerned. As a result there is poor attendance to clinic due to lack of support either financially or encouragement from the spouse. Findings also showed that males do not escort their pregnant partners due to a large age difference which is similar to a study done in Gambia by [21] which showed that a big age difference in a couple between an old man and a young woman restricts men from escorting their partners to a clinic as the men feel ashamed. Other reasons given for lack of involvement by men was their limited time availability for attending the clinic and this was further exacerbated by long waiting hours for clinic services and laboratory procedures. These findings are also similar to those reasons identified by [21] regarding the lack of male involvement in PMTCT programs.

Respondents $30 \%$ felt that there was much stigma surrounding HIV/AIDS and lack of sensitization to accept their status. This as a result leads to a larger percentage of the respondents not willing to disclose their status and as well as fear to uptake the available PMTCT services hence the underutilization of the services. There is disproportional relationship between the number of health care workers and the number of patients who come to seek care at the health facility. As a result the health care providers tend to be unfriendly due heavy workloads caused by high patient load and also because they are obliged to implement a multiple programme at the health facility and hence end up doing multiple activities. This concurs with the study finding [22] which confirmed unfriendly health care providers to be a barrier to uptake of PMTCT services.

Though a big number of the respondents $20 \%$ reported time spent waiting for antenatal services was too much, this may not stop them from coming for the services, but however wish the time was less. It was found that some of those who complained of the time being too much were public servants and selfemployed people who come for antenatal services and had to go to work, and would not wish to wait for long. Some of those who said the time was much come from very far places and need to walk back to their villages.

Some of the respondent $14 \%$ felt that they could not access the available PMTCT services as required due to financial shortages which they are facing due to high levels of poverty in their areas. Those who said so had to travel from far places in order to attend ANC and also to enroll on the PMTCT services at the clinic. Those who were not comfortable $58 \%$ with counseling reported that counseling was done for groups and not to individuals this makes them uncomfortable to ask personal questions. They preferred not to go for counseling at all and as a result they felt that they will be stigmatized if found reactive due to self-denial [23].

Most of the respondent 56\% were not willing to take PMTCT services, this was as a result of fear and escape from discrimination from the community if found reactive. They also noted that PMTC services were a waste of time since AIDS has no cure and they were sure that the virus will be definitely be transmitted to the infant. This was also attributed to lack of sensitization and sometimes no counseling done to the clients [22]. It was however noted that some respondents $38 \%$ reported not willing to take an HIV due to fear of being found positive. Most of the mothers $82 \%$ were not willing to disclose their HIV status after testing as this was a way of avoiding stigma from the community. Other even preferred not to take the test at the hospital laboratory since most of the workers were either neighbors or relatives. 
Texila International Journal of Public Health

Volume 5, Issue 2, May 2017

\section{Conclusion and recommendations}

\section{Conclusion}

The study concluded that both socio-cultural and structural factors play a role as barriers to the PMTCT programme and hence its outcomes. The study found that home deliveries are still common practice in a number of communities in Bungoma County and as a consequence impair the PMTCT programme. Lack of male involvement is another hindrance faced by the programme and men and the health facility have to find some measures to bring more men on board as ANC is not just a women only affair. Recruitment of new staff, review of current staff establishment and sending more health care workers for training and re-training are important measures for the programme as currently health facilities are facing a severe shortage of human resources and heavy patients load.

Health facilities still need a better way of monitoring their data as they rely only on manual data entries which most cases are likely to generate data of poor quality. Computerization of sites and employment of data clerks are required for proper data management. Many sites are trying to implement activities which would help to minimize and clear PMTCT barriers.

Other services such as linking of patients to support groups, defaulter tracing and conducting outreach activities to cater for patients who live far from the health facilities have been awarded to some of the sites but they need to be more emphasized and strengthened. People's attitude and utilization of PMTCT services were affected by the insufficient knowledge on what the PMTCT program entails and the benefits of the services as far as mother to child transmission of HIV is concerned.

Social demographic characteristics of the respondents, the knowledge and attitude towards PMTCT influenced their behavior towards PMTCT services.

\section{Recommendations}

The study wishes to recommend the following to the policy makers and all stake holders of the PMTCT and HIV/AIDS programs:

- Increase awareness of HIV/AIDS and PMTCT programs.

- Health education has to be provided by all key stakeholders and not only the hospital or health facilities. Dissemination of information has to be done at schools, churches, all gatherings, using media such as TV, radio, newspapers, etc.

- Programs should be multi-sectorial: have to involve not only health care workers but also committed politicians, village headmen, traditional herbalists and also traditional birth attendants.

- Clinic have to conduct outreach services to ensure they cover all pregnant mothers and to ensure that they are all registered for ANC services.

- Clinic have to motivate about male involvement and have to create an environment where men will feel a clinic is friendly towards them.

- Issues concerning HIV and stigma, as well as stigma associated with formula feeding, have to be cleared up through health education and disclosure.

- There is need to increase PMTCT counselors at the hospital staff to handle the PMTCT program in Bungoma county Referral hospital.

\section{References}

[1]. Agu, V. (2009). The Development of Harmonized minimum standards for guidance on HIV testing and counseling and prevention of mother to Child Transmission of HIV in the SADC region. PMTCT Country report. Namibia.

[2]. Babalola Stella and Adesegun Fatusi (2009) Determinants of use of maternal health services in Nigeria looking beyond individual and household factors. BMC Pregnancy and Childbirth 9:43

[3]. Chasela S Charles, Hudgens G Michael, Jamieson J Denise, Kayira Dumbani, Hosseinipour C Mina, Kourtis P Athena, Martinson Francis, Tegha Gerald, Knight J Rodney, Ahmed I Yusuf, Kamwendo D Deborah, Hoffman F Irving, Ellington R Sascha, Kacheche Zebrone, Soko Alice, Wiener B Jeffrey, Fiscus A Susan A, Kazembe Peter, 
Mofolo A Innocent, Chigwenembe Maggie, Sichali S Dorothy, van der Horst M Charles.(2010). Maternal or Infant Antiretroviral Drugs to Reduce HIV Transmission. N Engl J Med, 362 (24):2271-81.

[4]. Esther Ndonga, Zipporah Ng'ang'a, Erastus Muniu, Mohamed Karama and Martin Matu (2014) Barriers to Uptake and Effective Integration of PMTCT into SRH Services in Selected Health Facilities in Nairobi County, Kenya. Journal of Pediatrics \& Neonatal Care. Volume 1(4).

[5]. Fisher AA, Laing JE, Strocker J E (1998) Handbook for family planning, operation research design in sampling. Population Council. 40-45

[6]. George Awungafac, Patrick Achiangia Njukeng, Juliana Ajoache Ndasi, and Lawrence Tanyi Mbuagbaw (2015) Prevention of mother-to-child transmission of the Human Immunodeficiency Virus: investigating the uptake and utilization of maternal and child health services in Tiko health district, Cameroon. Pan Afr Med J. 2015; 20: 20

[7]. Gundel H, Katja S, Ilaria M, Chris B and Paulina M. (2005) Analyzing awareness and Handbook for family planning, operation research design in sampling. Population Immunodeficiency virus; a study on acceptability by Nigerian women attending clinics. African Journal of Reproductive Health.

[8]. Health Bridge. (2007). Challenges in the prevention of Mother to child transmission of HIV in Africa.

[9]. Katushabe J. (2006).Knowledge and attitude of pregnant women on the use of prevention of mother to child transmission of HIV (PMTCT) services in Mbale regional hospital.

[10]. Moth I A, Ayayo ABCO, Kaseje DO (2000).Assessment of utilization of PMTCT services at Nyanza Provincial Hospital, Kenya. Sahara J. PubMed. 2005;2:244-50. mother-to-child HIV transmission in resource-poor countries: Translating research into policy and practice. JAMA. PubMed, 283:1175-1182.

[11]. National AIDS and STI Control Programme (2009) Sentinel Surveillance of HIV in Antenatal Clinics 2008/2009. Minstry of Health, Nairobi, Kenya

[12]. NASCOP, "Kenya National Guidelines for HIV Prevention and Management of People Who Use Drugs (PWUD)", Kenya. 2013.

[13]. Perez, F., Mukotekwa, T, \& Miller, A.(2004). "Implementing a rural programme of prevention of mother-tochild transmission of HIV in Zimbabwe: first 18 months of experience." Tropical Medicine and International Health, vol. 9, no. 7, pp. 774-783.

[14]. Secka, E. (2010). Men's involvement in care and support during pregnancy and childbirth: A qualitative study conducted in Gambia.

[15]. Semrau K, Kuhn L, Vwalika C, Kasonde P, Sinkala M, Kankasa C, Shutes E, Aldrovandi G, Thea DM (2005) Women in couples antenatal HIV counseling and testing are not more likely to report adverse social events. AIDS. 19(6): 603-9.

[16]. Sirengo M, Muthoni L, Kellogg TA, Kim AA, Katana A (2014) Mother-to-child transmission of HIV in Kenya: results from a nationally representative study. J Acquir Immune Defic Syndr 66 Suppl 1S66-74.

[17]. UNAIDS 2010. Global Report. UNAIDS report on the global AIDS epidemic/2010. Geneva, Switzerland: Joint United Nations Programme on HIV/AIDS (UNAIDS); 2010.

[18]. UNAIDS (2013) UNAIDS report on the global AIDS epidemic 2013. Geneva

[19]. UNAIDS AIDS (2001) Declaration of Commitment on HIV/AIDS. United General assembly. Geneva.

[20]. UNICEF (2012) Tracking Porgress on child and maternal nutrition. Assessed from www.unicef.org/publications/files/Tracking_Progress_on_Child_and_Maternal_Nutrition_EN_110309.pdf, on 23 ${ }^{\text {rd }}$ February, 2015

[21]. USAID; (2001): Effective programs for preventing mother-To-Child HIV transmission. Washington DC. The population Council: Washington, DC USA

[22]. WHO (2013) Reproductive Health and Research. Assessed from

http://www.who.int/malaria/publications/atoz/iptp-sp-updated-policy-brief-24jan2014.pdf, on 23 ${ }^{\text {rd }}$ February, 2017.

[23]. Zolfo, M., Delvaux, T. \& Tamburrini, E. (2005). HIV/AIDS Knowledge and PMTCT Demand in Rural Zimbabwe. Washington: BMJ Publishing group 